\title{
Thermo-Mechanical and Morphological Properties of Water Hyacinth Reinforced Polypropylene Composites
}

\author{
Manabendra Saha and Ali Md. Afsar

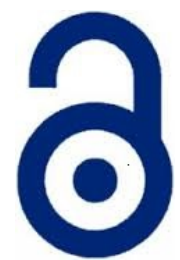 \\ Received: 01 August 2018 \\ Accepted: 08 September 2018 \\ Published: 15 September 2018 \\ Publisher: Deer Hill Publications \\ (c) 2018 The Author(s) \\ Creative Commons: CC BY 4.0
}

\section{ABSTRACT}

This paper focusses on the analysis of thermo-mechanical and morphological properties of water hyacinth (WH) fiber reinforced polypropylene (PP) biocomposites manufactured by using a single screw extruder and an injection molding machine. With a view to increasing the compatibility between the WH fibers and polypropylene matrix, raw WH fibers were chemically treated with Benzenediazonium salt in base media. Composites were manufactured with five different levels of loading $(15,20,25,30$ and $35 \mathrm{wt} \%)$ of both the raw and treated WH fibers. Thermal properties of WH-PP composites were evaluated by thermogravimetric and differential thermal analyses. To analyze mechanical properties of composites, tests of tensile strength and stiffness, flexural strength and stiffness, and Charpy impact strength were carried out following ASTM standards. It was found that thermal stability and all the mechanical properties except tensile strength were improved considerably for chemically treated WH fiber composites in comparison with untreated ones. Fracture surfaces of the tensile and flexural specimens were scanned with scanning electron microscopy (SEM) to understand their surface morphologies. The SEM images clearly revealed that there were fewer fiber agglomerations, microvoids, and fiber pull out traces in treated WH-PP composites than in the untreated ones indicating better distribution of the fibers into the matrix as well as stronger fiber matrix interfacial adhesion due to treatment of WH fibers. Water absorption properties were studied to evaluate the viability of these biocomposites under specified conditions.
\end{abstract}

Keywords. Thermo-mechanical properties, scanning electron microscopy, water hyacinth fiber, polypropylene, biocomposites

\section{INTRODUCTION}

Natural fiber reinforced polymer matrix composites, often called biocomposites, are now preferred over artificial fiber reinforced composites as natural fiber composites have outstanding advantages of environment friendliness, biodegradability, recyclability, cost-effectiveness, and better physico-mechanical properties [1-6]. The using of biobased lignocellulosic natural fibers as reinforcing materials for manufacturing of biocomposites is gaining more and more approval day by day due to their flexibility during processing (require low processing temperature), higher specific stiffness, lightweight, non toxic properties, and low cost. So far, numerous researches have been carried out on the polymer composites [7-13], where different natural fibers such as wood fibers and flour, kenaf fibers, sago, rice starch, corn starch, pineapple leaf fibers, and coir fibers were used as fillers in polymer matrices. Among various natural fibers easily available to human beings, water hyacinth (Eichhornia crassipes) is one of the cheapest fibers and quite new in reinforcing thermoplastics. An experimental study to evaluate the mechanical and thermal properties of water hyacinth (WH) fiber reinforced low density polyethylene (LDPE) composites was conducted by Supri et al.[14]. This study appears to be the first attempt of using WH fibers as a reinforcing material. They observed good mechanical properties of WH-LDPE composites lead to concluded that WH fibers have a great potential to use as a filler materials

\section{Saha ${ }^{1,2} \otimes$ and A. M. Afsar ${ }^{2}$}

1 School of Mechanical Engineering

The University of Adelaide, SA 5005, Australia

E-mail: manabendra.saha@adelaide.edu.au,

2Department of Mechanical Engineering

Bangladesh University of Engineering and Technology

Dhaka 1000, Bangladesh

E-mail: mdafsarali@me.buet.ac.bd

Reference: Saha, M., and Afsar, A. M. (2018). Thermo-mechanical and Morphological Properties of Water Hyacinth Reinforced Polypropylene Composites. International Journal of Engineering Materials and Manufacture, 3(3), 151-161. 
in polymeric composites owing to their high content of cellulose. However, no systematic studies have been conducted for better understanding the thermal and mechanical properties of WH fiber reinforced polypropylene (PP) composites. WH is abundant in nature and reproduces freely on the surface of fresh water and also has a minimal effect on the environment due to their biodegradable properties [8]. Therefore, it is necessary to systemically explore the thermo-mechanical and morphological properties of WH-PP biocomposites to know their potential applications as engineering materials.

The physico-mechanical properties of lignocellulosic biocomposites mostly depend on the type of matrix, the properties and volume fraction of the reinforcing fibers, and fiber-matrix interaction. Uniform dispersion of the reinforcements can be achieved by effective mixing of the components and a proper compounding process. Like other natural fibers, one shortcoming that restricts the extensive usage of the WH fibers is the lack of good adhesion with most polymeric matrices [8, 14, 15]. The hydrophilic nature of WH fibers adversely affects adhesion with hydrophobic polypropylene (PP) matrix that may cause a loss of strength. To get rid of this problem, the surfaces of WH fibers have to be modified by suitable chemical treatment in order to promote adhesion.

It is reported [6] that the compatibility between fibers and matrix materials can be improved to a large extent by chemical treatments of the lignocellulosic fibers using a suitable chemical agents [6]. The effect of chemical treatment on coir fibers with benzenediazonium salt was analysed by Haque et al. [16]. Coir fibers were seen to have immense potential of better compatibility with PP after chemical modification and consequently the mechanical properties of the composites were increased significantly. Edeerozey et al. [6] reported that alkalization treatment of kenaf fibers with $\mathrm{NaOH}$ had improved significantly the mechanical properties of the kenaf fiber reinforced composites as compared to that of untreated fibers. Islam et al. [7] investigated the effect of chemical pre-treatment of wood fibers with $5 \%$ alkaline $\mathrm{NaOH}$ solution on physical and mechanical properties of wood polymer composites. They found that the chemical modification improved overall properties of the composites compared to that of the untreated fiber reinforced composites. This improvement was attributed to the better adhesion between polymer matrix and the chemically treated wood fibers. In the present paper, raw WH fibers were chemically treated with benzenediazonium salt in alkaline medium to increase the compatibility and adhesion of the WH fibers with the PP matrix. Composites were manufactured with both the treated and raw WH fibers and PP by using a single screw extruder and an injection molding machine. The thermo-mechanical and surface morphological properties of the WH-PP composites are presented. Also, the effect of the fiber loading on the above properties of the WH-PP composites are analysed and discussed.

\section{EXPERIMENTAL}

\subsection{Materials}

The thermoplastic polypropylene (PP) used as matrix material was supplied by the Linyi Aosen Chemicals Co. Ltd., Shandong, China, in the form of homo-polymer pellets. According to the manufacturer, the melt flow rate (MFR) of $8 \mathrm{~g} / 10 \mathrm{~min}$ and melting temperature of $165-171{ }^{\circ} \mathrm{C}$ were noted for the specified PP. The fresh water hyacinth fiber was collected from local area of Bangladesh. The stems of the fresh water hyacinth were cleaned to remove adhering dirt and cut into small pieces followed by drying under solar radiation for 48 hours to evaporate any trace of water content. The dried water hyacinth was ground into powder, named as water hyacinth (WH) fibers. It comprises $18.4 \%$ cellulose, $49.2 \%$ hemicellulose, $3.55 \%$ lignin, $12.60 \%$ crude protein, and $16.25 \%$ other constituents [17]. The measured diameter of the WH fibers was between 350 to $400 \mu \mathrm{m}$. Chemicals used in this study to treat WH fibers were hydrochloric acid $(\mathrm{HCl})$, sodium nitrite $\left(\mathrm{NaNO}_{2}\right)$, sodium hydroxide $(\mathrm{NaOH})$, aniline $\left(\mathrm{C}_{6} \mathrm{H}_{5} \mathrm{NH}_{2}\right)$, and $\mathrm{N}, \mathrm{N}$ dimethyle aniline $\mathrm{C}_{6} \mathrm{H}_{5} \mathrm{~N}\left(\mathrm{CH}_{3}\right)_{2}$.

\subsection{Chemical Treatment of WH Fiber}

In an attempt to improve mechanical properties of the composites, WH fibers were chemically treated using benzenediazonium salt. The hydroxyl group in the raw WH fibers is responsible for high water absorption and weak interfacial bond between the fibers and PP matrix. The chemical treatment converts the hydroxyl groups into diazo group and results in azo product, 2,6-diazocellulose. Benzenediazonium salt was synthesized by the standard diazotization method using both aniline and N,N-dimethyle aniline (NNDMA). WH fibers were dried at $105^{\circ} \mathrm{C}$ for $24 \mathrm{hr}$ in order to minimize its moisture content to $1-2 \%$ and then kept in a sealed container. One litter $5 \% \mathrm{NaOH}$ solution was taken in a 5 litter beaker into which $750 \mathrm{~g} \mathrm{WH}$ fibers were submerged and kept for 10 min at about $5^{\circ} \mathrm{C}$ in an ice bath. A freshly prepared cooled (temperature below $5^{\circ} \mathrm{C}$ ) solution of benzenediazonium salt was then poured slowly into the above mixture with constant stirring and kept for about $10 \mathrm{~min}$. Later, the fibers were washed properly with distilled water and dried in open air under sunlight for 36 hours. WH fibers were treated with two types of Benzenediazonium chloride: (Anl)/ $\mathrm{NaOH}$ and (NNDMA)/NaOH.

\subsection{Fabrication of Composites}

WH fibers were weighed according to the required weight fraction of fibers. Then they were dried in an oven at a temperature slightly higher than $100{ }^{\circ} \mathrm{C}$ for about $1 \mathrm{hr}$ to evaporate any trace of moisture. Weighed amount of commercial polypropylene was taken in a beaker. To prevent voids, water bubbles, poor fiber-matrix adhesion, the polypropylene was dried in an oven at about $100{ }^{\circ} \mathrm{C}$ for $3 \mathrm{hr}$ [4]. WH fibers were manually mixed thoroughly with PP granule in the beaker at various weight proportions. The WH fibers and PP were then melt-mixed by passing them 
through a single screw extruder machine. The processing temperature of the extruder was controlled at $165^{\circ} \mathrm{C}$. The melt-mixed WH-PP was delivered from the extruder in the form of a rod of small diameter. The rod shaped meltmixed WH-PP was cut into small pieces of 5-10 mm length by a scissor. The small pieces of melt-mixed WH-PP products were then fed into an injection molding machine in order to prepare composite specimens directly as per ASTM standard. The injection molding temperature was maintained at $165^{\circ} \mathrm{C}$. The injection-molding machine was vertical and operated manually. The tensile test specimens and bending test specimens were fabricated by using corresponding die in the injection molding machine. Finally, the specimens were carefully discharged from the die after complete cooling.

\subsection{Thermal Property}

The thermogravimetric analysis (TCA) and derivative thermal gravimetric (DTC) analysis were conducted by a TG/DTA-630 module analyser. The samples were scanned from $25^{\circ} \mathrm{C}$ to $600^{\circ} \mathrm{C}$ at $20^{\circ} \mathrm{C} / \mathrm{min}$ in the presence of nitrogen.

\subsection{Mechanical Property}

In order to investigate the mechanical properties of the WH-PP composites, tensile, three point bending, and Charpy impact tests were carried out. The static tensile test of the composites were carried out in an universal tensile testing machine (Model Hounsfield UTM (H10KS), capacity: 10KN, Ogawa Seiki C. Ltd., Japan) at a cross head speed of 5 $\mathrm{mm} / \mathrm{min}$. Tensile tests were carried out following ASTM D 638-01, 2002 standard. The tensile strength and Young's modulus were calculated automatically by the QMAT software. Three point bending tests were carried out according to ASTM D 790-00, 2002 using the same testing machine mentioned above at the same crosshead speed. The dynamic Charpy impact tests were conducted according to ASTM D 6110-97, 2002 using an universal impact testing machine (Type: TIT-30, Tokyo Testing Machine MFC. Co. Ltd., Japan). Weight of the hammer of this machine was $20.54 \mathrm{~kg}$. Notched composite specimens were used during the experiment.

\subsection{Water Absorption property}

The water absorption tests of the composites were carried out following ASTM: D 570-98, 2002. To measure the water uptake capacity of the composites, rectangular specimen of dimension76 mm $\times 25 \mathrm{~mm} \times 3.4 \mathrm{~mm}$ was prepared. From the difference of the final and initial weights before and after immersion in a water bath for $24 \mathrm{hr}$, percentage of water uptake was calculated. In each test and type of composite, 10 specimens were tested and the average values are reported.

\subsection{Morphological Properties}

The morphology of the fracture surfaces of composites were studied by using scanning electron microscope (SEM), Model: JSM-5510 SEM analyser from JEOL Co. Ltd., Japan. Since the WH-PP composites are not conductive, before the examination of SEM, the fractured samples were mounted on aluminium stubs and allowed to undergo sputtering coating to make the samples conductive. The sample surfaces were sputter-coated with a thin gold layer of $20 \mathrm{~nm}$ to avoid electrostatic charging during examination.

\section{RESULTS AND DISCUSSIONS \\ 3.1 Thermal Properties}

Thermal characteristics of raw and treated WH fiber reinforced composites are displayed in Figs. 1 and 2. The DTC and TGA have been carried out to evaluate the thermal stability and degradation of $35 \mathrm{wt} \% \mathrm{WH}$-PP composites. The DTG curves (Fig.1) of all composites showed an initial peak between $260{ }^{\circ} \mathrm{C}$ and $350{ }^{\circ} \mathrm{C}$ which corresponds to the thermal decomposition of hemicelluloses and the glycoside linkage of cellulose. After this peak, the curve corresponding to Benzenediazonium salt (NNDMA)/ $\mathrm{NaOH}$ treated WH-PP composite exhibits single peak at $469{ }^{\circ} \mathrm{C}$ temperature while the raw WH-PP composite exhibits the peak at $462{ }^{\circ} \mathrm{C}$ temperature. The DTC curve of Benzenediazonium salt $(\mathrm{Anl}) / \mathrm{NaOH}$ treated WH-PP composite exhibits single decomposition with the highest peak at decomposition temperature of $478^{\circ} \mathrm{C}$. The peak in the range of $400-500^{\circ} \mathrm{C}$ is due to the thermal decomposition of lignin and $\alpha$ - cellulose [9].

Figure 2 shows the results of thermal gravimetric analysis (TCA) of WH-PP composites with 35\% fiber loading. Table 1 shows the temperature of 50\% weight loss, the final decomposition temperature, and residual mass of raw and treated WH-PP composites. These results show that Benzenediazonium salt, $(\mathrm{Anl}) / \mathrm{NaOH}$, treated WH-PP composites exhibit more thermal stability than those of Benzenediazonium salt, (NNDMA)/NaOH, treated WH-PP composites and raw WH-PP composites in terms of the highest final decomposition temperature and largest amount of residual mass. The raw WH-PP composites have shown least thermal stability. This was due to the presence of good interfacial adhesion between chemically modified WH fibers and PP as a result of uniform dispersion of fiber throughout the PP matrix. The better dispersion of filler acts as a barrier against the release of volatile matter from the composites during the thermal degradation. It also might be attributed to an adsorption effect of volatile gases at the fiber surface which affects to slow down the decomposition rate of polymer composites[18]. 
Table 1: Decomposition temperature of 35 wt\% WH-PP composites

\begin{tabular}{llllll}
\hline Composites & \multicolumn{2}{l}{ Derivative thermal gravimetric (DTG) analysis } & \multicolumn{2}{c}{ Thermogravimetric analysis (TCA) } \\
\hline & $\begin{array}{l}\text { Decomposition } \\
\text { beginning temp. } \\
\left({ }^{\circ} \mathrm{C}\right)\end{array}$ & $\begin{array}{l}\text { Decomposition peak } \\
\text { temp. }\left({ }^{\circ} \mathrm{C}\right)\end{array}$ & $\begin{array}{l}\mathrm{T}_{\text {-50 }} \text { \% wt } \\
\left({ }^{\circ} \mathrm{C}\right)\end{array}$ & $\begin{array}{l}\text { Final } \\
\text { decomposition } \\
\text { temp. }\left({ }^{\circ} \mathrm{C}\right)\end{array}$ & $\begin{array}{l}\text { Residual mass } \\
(\%)\end{array}$ \\
\hline $\begin{array}{l}\text { Raw WH-PP } \\
\text { NNDMA/NaOH }\end{array}$ & 269 & 462 & 453 & 597 & 3.09 \\
$\begin{array}{l}\text { treated WH-PP } \\
\text { Anl/NaOH treated }\end{array}$ & 329 & 469 & 458 & 598.5 & 5.59 \\
WH-PP & 345 & 478 & 472 & 598.5 & 9.65 \\
\hline
\end{tabular}

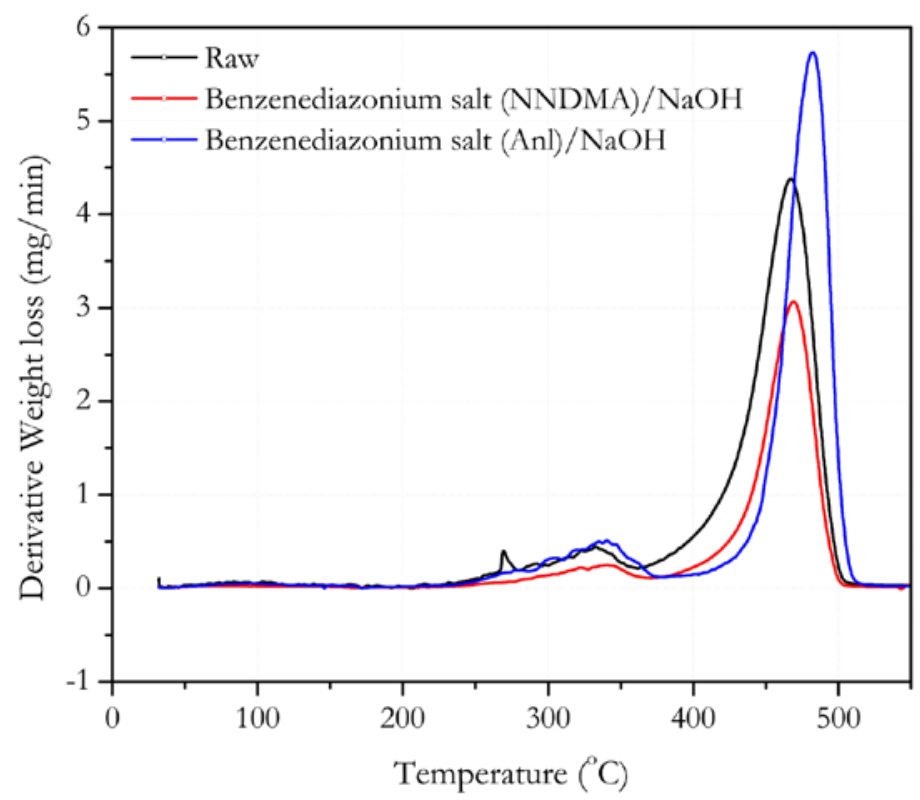

Figure 1: Derivative weight loss of 35 wt\% WH-PP composites as a function of temperature

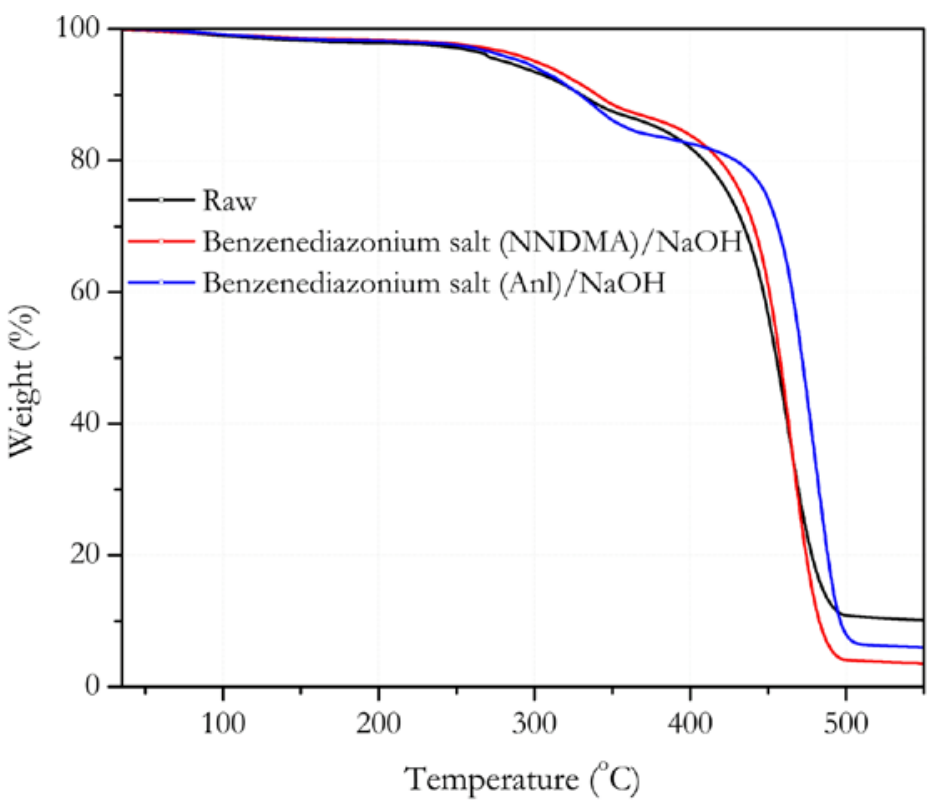

Figure 2: Weight of $35 \mathrm{wt} \% \mathrm{WH}-\mathrm{PP}$ composites as a function of temperature 


\subsection{Mechanical Properties \\ 3.2.1 Tensile Properties}

The tensile strengths of the raw and chemically treated WH-PP composites at different fiber loadings are shown in Fig. 3. It is observed from the figure that the tensile strength of the WH-PP composites decreased with fiber loading. Similar results were also reported by other researchers [4, 12-14, 18-21]. WH fibers are hydrophilic in nature owing to the presence of hydroxyl groups of the cellulose of WH, whereas the nature of PP is hydrophobic. Thus, the hydrophilic WH fiber does not interact well with the hydrophobic PP. As a consequence, the weak interfacial area between the WH fiber and PP matrix was increased with the increment of fiber loading that contributes to the decreasing trend of tensile strength. In order to increase the compatibility of the WH fibers with PP matrix, raw WH fibers were chemically treated with $(\mathrm{Anl}) / \mathrm{NaOH}$ and $(\mathrm{NNDMA}) / \mathrm{NaOH}$. This consequently increased the tensile strength of $(\mathrm{Anl}) / \mathrm{NaOH}$ treated WH-PP composites. It is found that the tensile strength increased approximately 4$12 \%$ over the raw WH fiber composites. The overall change of tensile strength due to the effect of chemical treatment may be due to the change in the structure of the cellulose unit of $\mathrm{WH}$. The chemical treatment of $\mathrm{WH}$ reduced the hydroxyl group of the cellulose unit by coupling with basic diazonium salt. However, tensile strength of (NNDMA)/NaOH treated WH-PP composites decreased slightly over the raw WH-PP composites. The reason behind the decrease of tensile strength in this case is not well understood. The chemical reaction pathway is expected to be more complex than that of previous case, however the decreasing trend of tensile strength of (NNDMA)/ $\mathrm{NaOH}$ treated WH-PP composites with fiber loading is not dissimilar of raw or (Anl)/NaOH treated WH-PP composites.

However, in all the cases of composites, the strength is lower than that of neat PP. Figure 4 exhibits the Young's modulus of the composites corresponding to different fiber loadings. As expected, the addition of fiber increases the modulus of the composites, resulting from the inclusion of rigid fiber into the soft PP. This observation suggests that the incorporation of rigid fibers into the soft thermoplastic PP increases the stiffness of the composite. The chemically treated WH-PP composites are found to show higher modulus compared to those of the untreated ones. This indicates that homogeneous dispersion of WH fibers and better fiber-matrix interaction can be achieved by treatment of WH fibers. The range of the Young's modulus found in the present research is between 608-761 MPa, which is significantly higher than that obtained in a previous research [15] (150-260 MPa) using the same fiber material. The higher values of the Young's modulus found in the current research might be due to the chemical treatment of WH fiber and use of different matrix material. Between the two treated fiber composites, (Anl)/ NaOH treated WH-PP composite appears to be better in terms of tensile strength and modulus than those of (NNDMA)/ $\mathrm{NaOH}$ treated WH-PP composite.

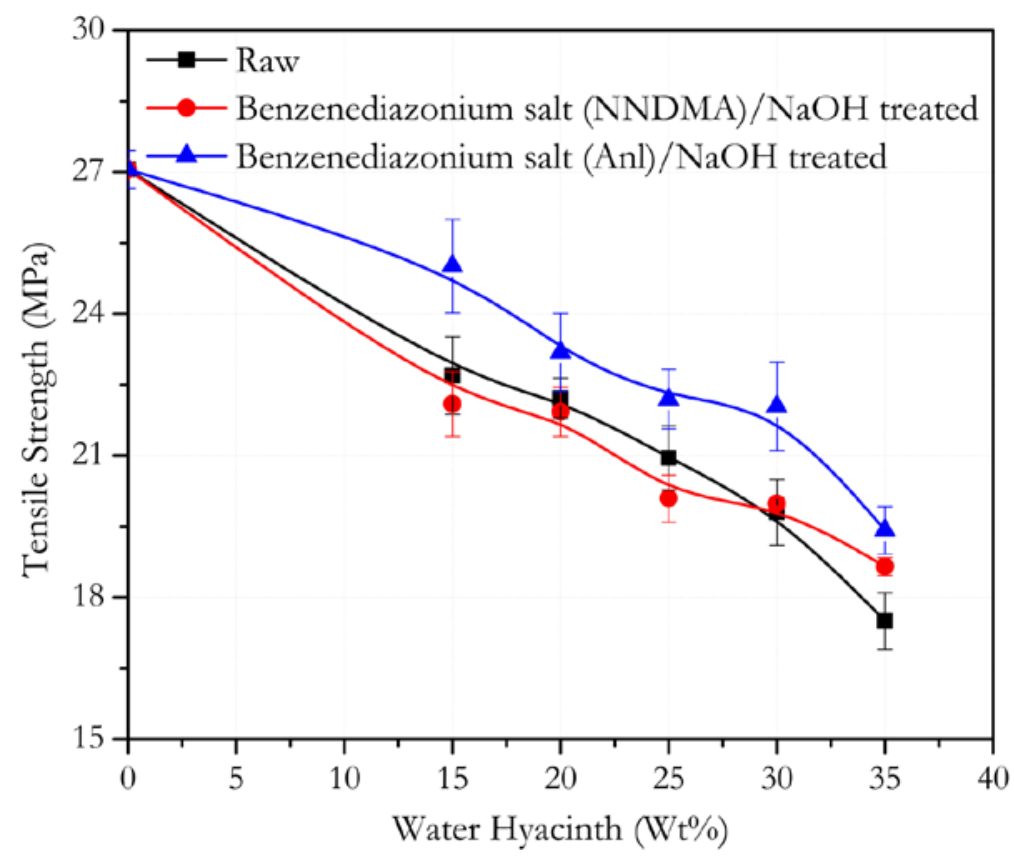

Figure 3: Effect of fiber loading on tensile strength of WH-PP composites

\subsubsection{Flexural Properties}

The variation of the flexural strength and modulus of both raw and treated WH reinforced PP biocomposites at different fiber loadings is shown in Figs. 5 and 6, respectively. From Fig. 5, it is seen that the flexural strength of raw WH-PP composite increased with fiber loading up to $25 \%$ followed by reduction of strength for further addition of fibers. On the other hand, for chemically treated WH fiber reinforced PP composites, flexural strength increased 
steadily with the increase of the fiber content. The steady behaviour of flexural strength of the treated WH-PP composites could be a balance in the favourable entanglement of the polymer chain with the filler and opposing weak filler-matrix interfacial adhesion with increasing filler content. The (NNDMA)/ $\mathrm{NaOH}$ treated WH-PP composites yielded higher flexural strength compared to the other WH-PP composites. It is found that the flexural strength increased by approximately $10-28 \%$ over the raw WH fiber composites, while the increment was 4-11\% over (Anl)/ $\mathrm{NaOH}$ treated WH-PP composites. It is evident from Fig. 6 that the addition of WH fibers to PP has significantly increased the modulus of the composites, which is found to be in good agreement with the results of previous studies $[8,14,15]$. Since $\mathrm{WH}$ fiber is a high modulus material [14], higher fiber concentration in the composites demands stronger stress for the same amount of deformation. Consequently, flexural modulus of the composites increases with an increase in the fiber content. Both the flexural strength and modulus are found to be higher for chemically treated fiber, which could be due to better fiber-matrix interfacial adhesion and effective stress transfer from the matrix to the fiber.

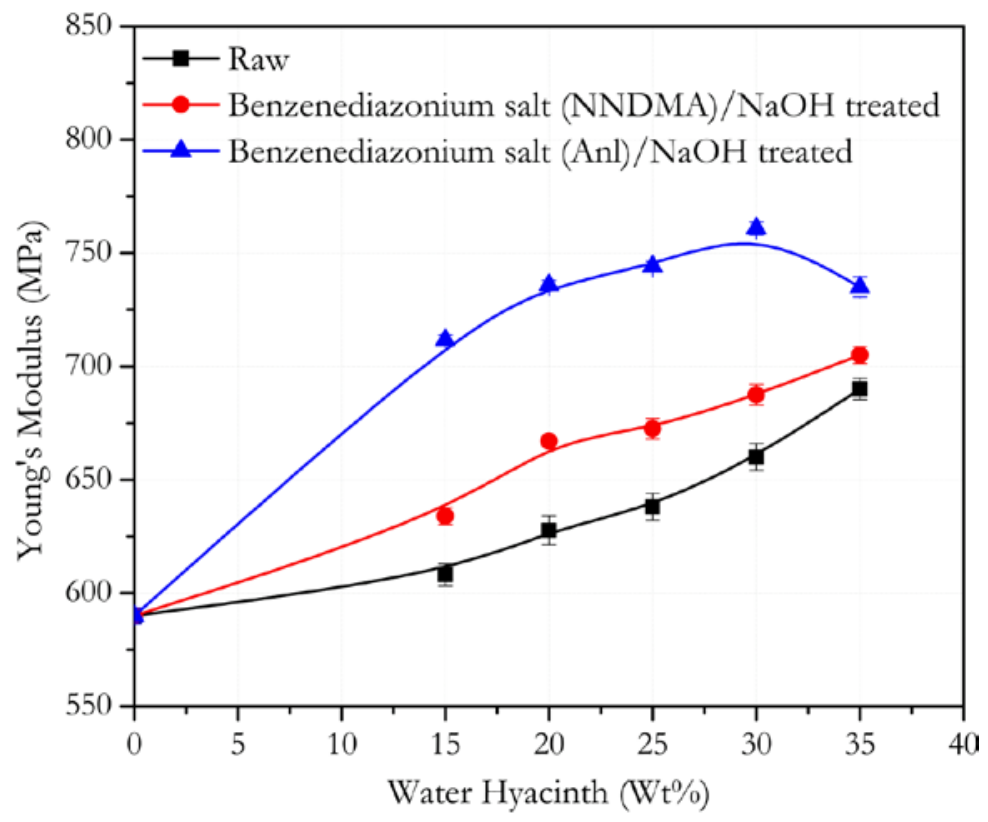

Figure 4: Effect of fiber loading on the Young's modulus of WH-PP composites

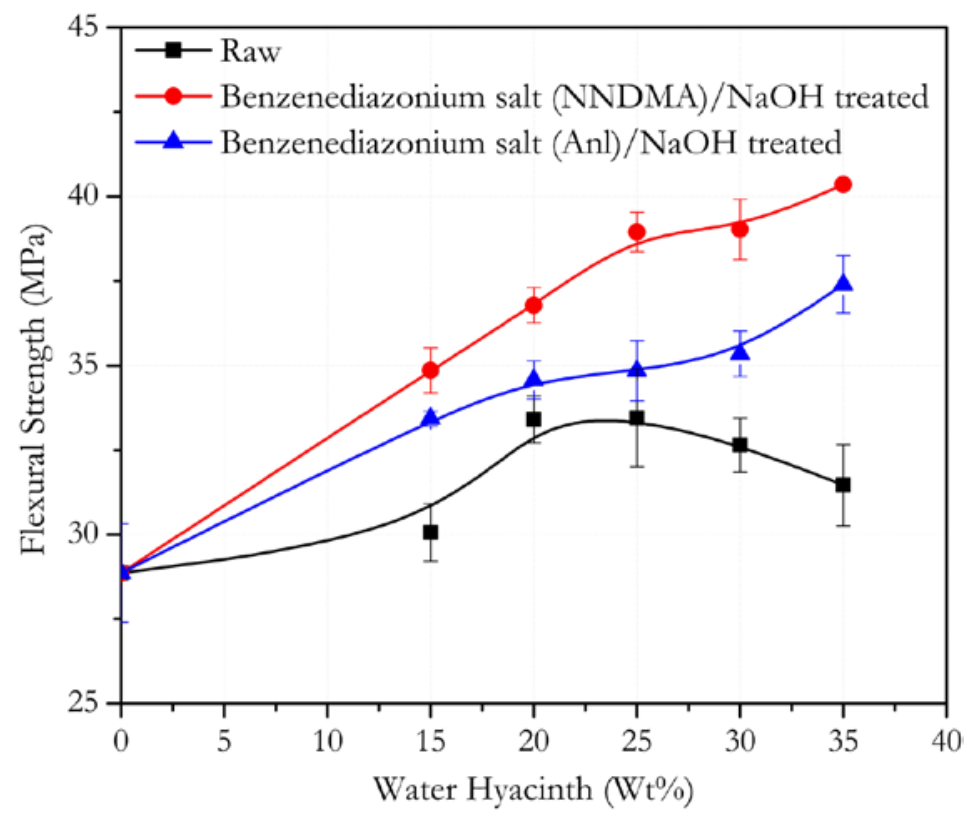

Figure 5: Flexural strength as function of fiber loading in WH-PP composites 


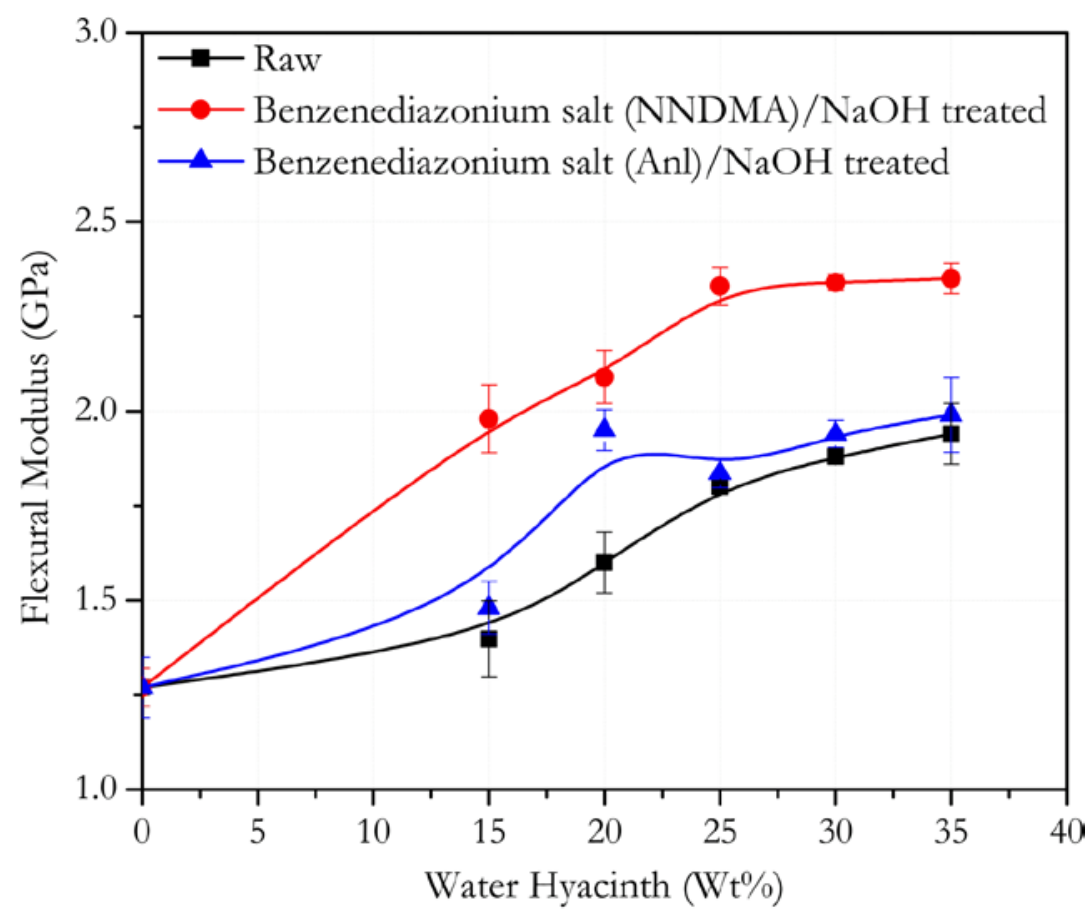

Figure 6: Flexural modulus as a function of fiber loading in WH-PP composites

\subsubsection{Impact Strength}

Charpy impact strength as a function of fiber content of both the raw and chemically treated WH fiber reinforced PP composites is shown in Fig. 7. The Charpy impact strength test is a standardized high strain-rate test which determines the amount of energy absorbed by a material during fracture. This absorbed energy is a measure of a given material's toughness and acts as a tool to study brittle-ductile transition. For fiber-reinforced polymeric composites, it depends on a number of factors, such as the nature of the fiber, polymer matrix, and the polymer-matrix interfacial bonding. An increase in toughness, i.e., increase in the resistance of crack propagation occurs if fiber bridges the crack in the composites [4, 19]. As shown in Fig. 7, impact strength of both the raw and treated WH- PP composites show an increasing trend with an increase in the fiber loading, implying that the fiber is capable of absorbing energy. Further, treated WH-PP composites have improved impact strength over untreated WH-PP composites. This ensures the improved interfacial bonding between fibers and matrix providing an effective resistance to crack propagation during impact tests. Again, (NNDMA)/NaOH treated WH-PP composites exhibit higher impact strength than those of (Anl)/ $\mathrm{NaOH}$ treated composites. It is found that the impact strength of (NNDMA)/NaOH treated WH-PP composites increased by approximately $31-78 \%$ over the raw WH fiber composites, while the increment was $25-50 \%$ over (Anl)/ $\mathrm{NaOH}$ treated WH-PP composites. Significantly higher impact strength for (NNDMA)/NaOH treated WH-PP composites is probably due to the favourable interaction between the treated WH fiber and the hydrophobic PP chain of the matrix. It is speculated that the so-called fiber pullout and fiber agglomeration could be responsible for the lower impact strength of raw WH-PP composites.

\subsubsection{Water Absorption Characteristics}

Water absorption of WH fiber reinforced PP composites is one of the important characteristics that determines the suitability of terminal applications of these composites. Water absorption could lead to a decrease in some of the properties and needs to be considered when selected for a specific application. In fact, it is difficult to eliminate entirely the absorption of moisture from the composites without using expensive surface coatings on the surface of composites and/or fibers. Water absorption in lignocellulosic fiber-enriched composites can lead to build up of moisture in the fiber cell wall and also in the fiber-matrix interphase region. Water absorption characteristics of the WH-PP composites against fiber loading are shown in Fig. 8. It shows that water absorption increased with an increase in fiber loading. Usually natural fiber-polymer composites without compatibilizer show remarkable water absorption due to the presence of voids [4, 11, 16, 21-23]. With an increase in fiber loading, the number of hydroxyl groups as well as microvoids in the composites increases, which result in an increase in water absorption. However, chemically treated WH fiber reinforced composites of the present study are found to show lower water absorption tendency compared to the untreated ones, indicating that the hydrophilic nature of WH fiber has substantially decreased upon chemical treatment with Benzenediazonium salt. This can directly be attributed to the decrease in the number of 
hydroxyl groups responsible for the hydrophilic nature of the cellulose that converted into diazo group and results in azo product, 2,6-diazocellulose. No dimensional change was observed upon immersion of the composites in water. This indicates that fibers are thoroughly encapsulated in the matrix. At the same time, it can also be ascribed that, due to favourable interaction between the matrix and the treated fibers, microvoids in the composites have substantially minimized, exhibiting lower water uptake tendency.

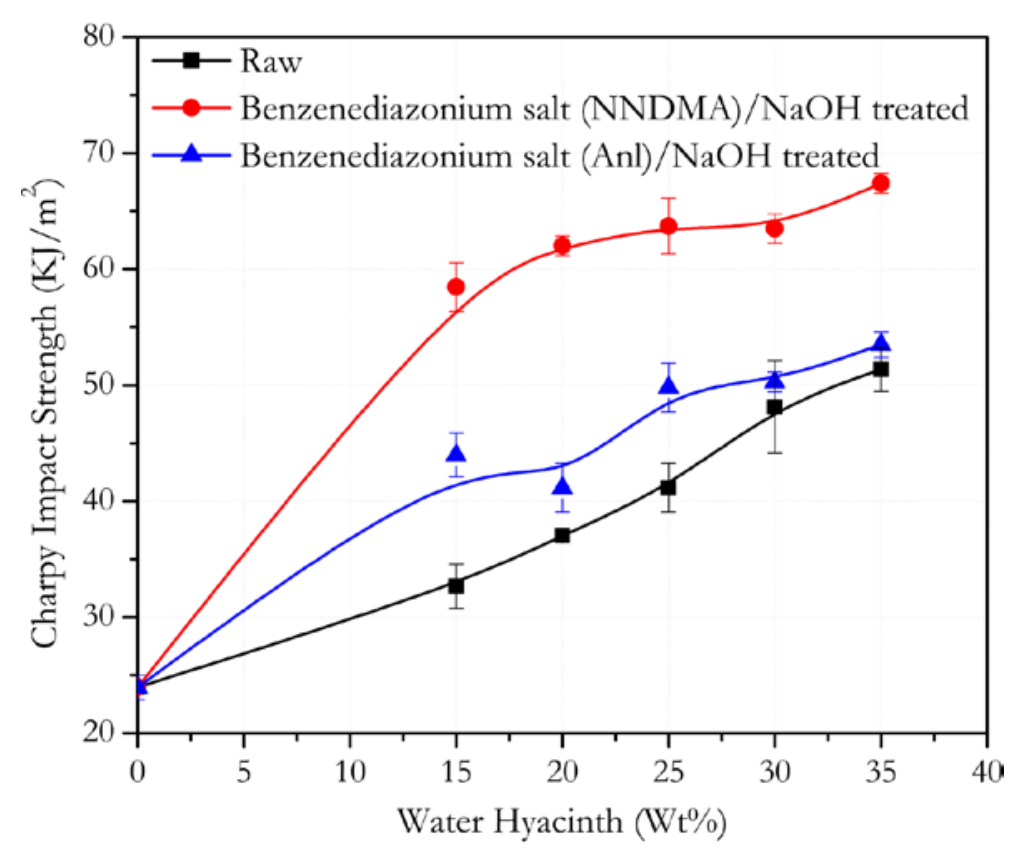

Figure 7: Impact strength versus fiber loading curves of WH-PP composites

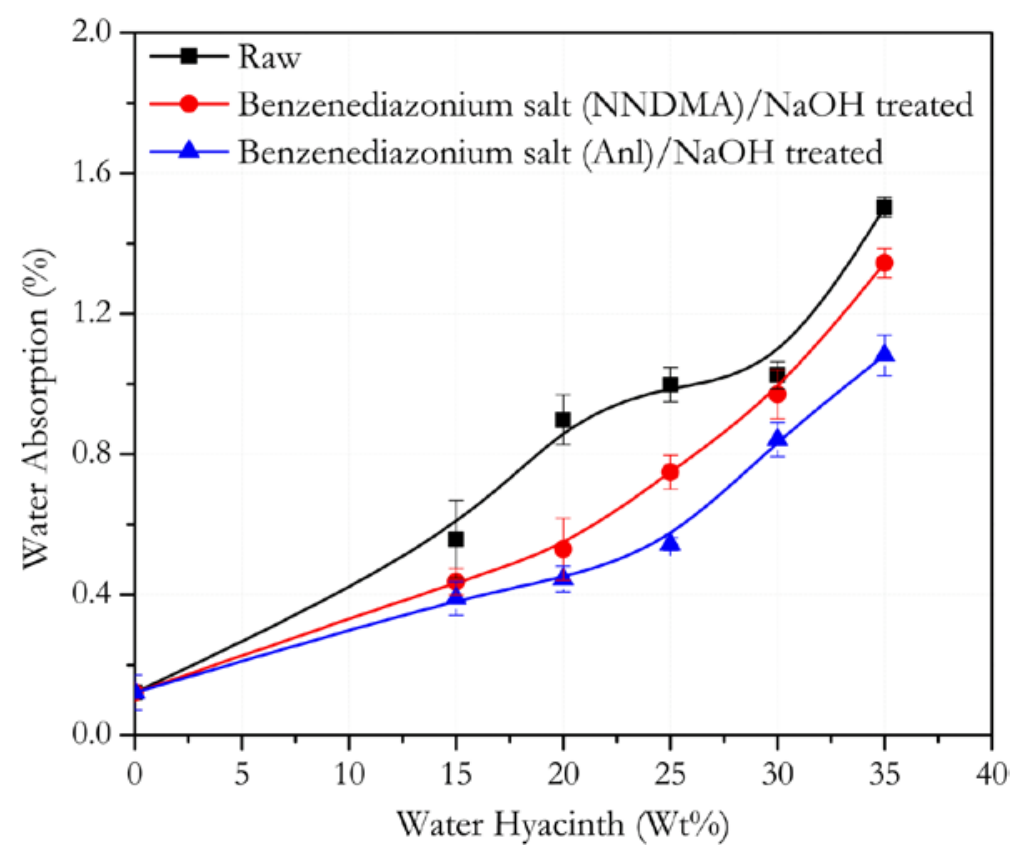

Figure 8: Water absorption characteristics of WH-PP composites 


\subsection{Morphological Properties}

The surface morphology of tensile and flexural fracture surfaces reflects the reasons why mechanical properties of composites prepared from fibers of different surface properties are different. The morphological features of tensile and flexural fracture surfaces of $35 \mathrm{wt} \% \mathrm{WH}$ fiber reinforced PP biocomposites investigated by SEM are shown in Fig. 9. Figures 9(a) - (c) represent the fracture surfaces of tensile specimens while Figs. 9(d) - (f) represent the fracture surfaces of flexural specimens. It can be easily traced out that there are a number of agglomerations resulting from strong fiber-fiber interaction and fiber pullout marks in the SEM images of the raw WH fiber reinforced PP composite (Fig. 9a). This implies that there is poor interfacial bonding between the matrix and the raw fibers. The raw WH fibers can be clearly seen in the composite micrograph of Fig. (9)d due to fiber pullout as a result of weak interfacial bonding between the eaw fiber and matrix.. For this reason, the raw WH-PP composites had worst set of mechanical properties. From SEM images of chemically treated composites (Figs. 9(b), (c), (e), and (f)), it is seen that the fibers and matrix are not clearly differentiable due to improved interfacial bonding between them. During chemical treatment, hydrophilic - $\mathrm{OH}$ groups in the raw WH cellulose were converted to hydrophobic groups. Consequently, the interaction and interfacial bonding between the WH fibers and the PP matrix was increased, which in turn, increased the physico-mechanical properties of the resultant composites. Also, SEM images of chemically treated WHPP composites show almost no signs of fiber agglomeration and microvoids in the fracture surface of the composite. In addition, the pullout traces are found to have substantially decreased for treated WH-PP composites. This supports that the interfacial adhesion between the chemically treated $\mathrm{WH}$ fiber and the PP matrix has become much more favourable upon treatment of the fibers.
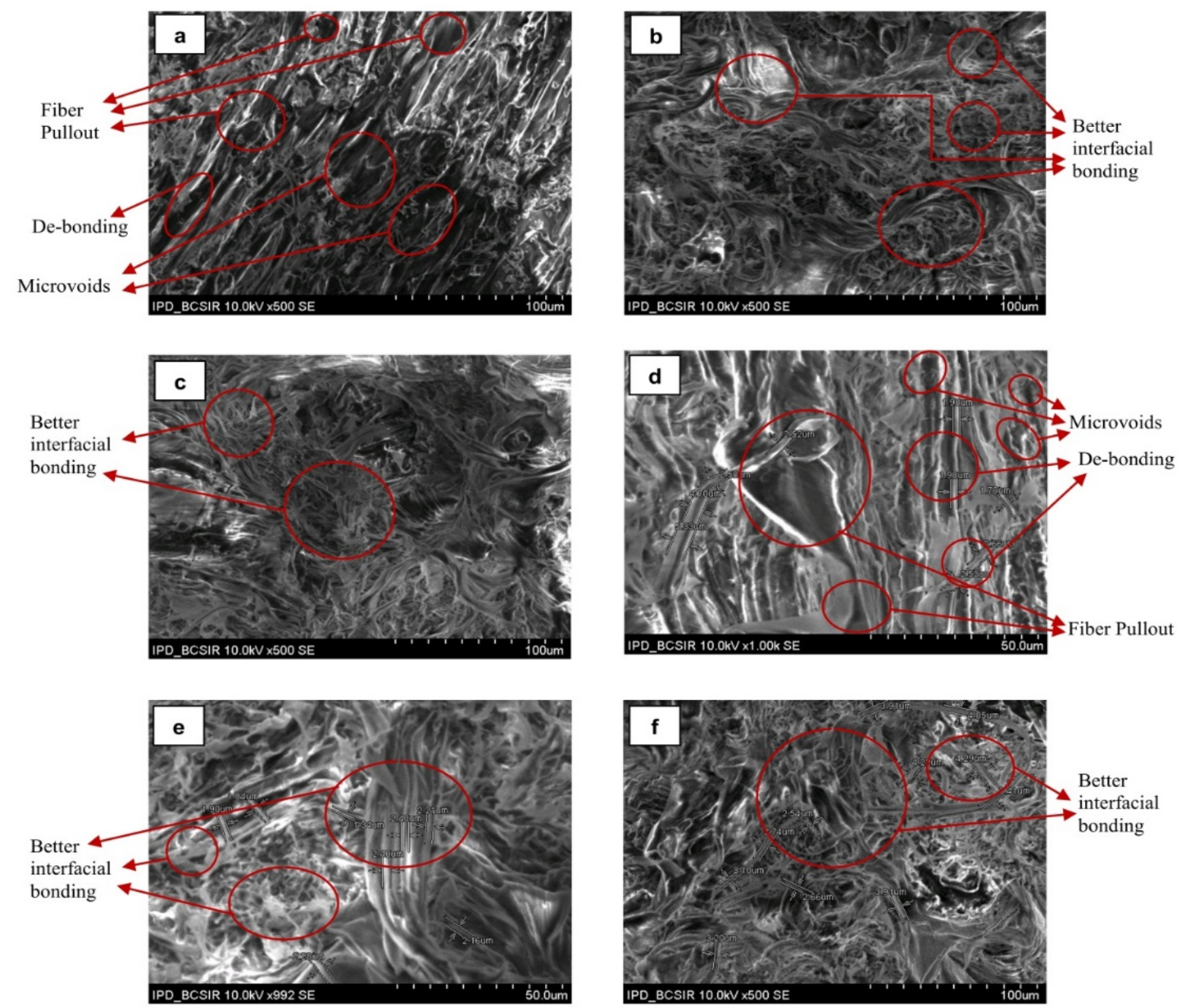

Figure 9: SEM micrographs of $35 \mathrm{wt} \% \mathrm{WH}-\mathrm{PP}$ composites: (a) tensile fracture surface of raw WH composite, b) tensile fracture surface of (NNDMA)/NaOH treated WH composite, c) tensile fracture surface of (Anl)/ $\mathrm{NaOH}$ treated WH composite, (d) flexural fracture surface of raw WH composite, (e) flexural fracture surface of (NNDMA)/NaOH treated WH composite, and (f) flexural fracture surface of (Anl)/ NaOH treated WH composite. 


\section{CONCLUSIONS}

The effects of fiber loading and chemical treatment of WH fibers on the thermo-mechanical and morphological properties on WH-PP composites have been investigated. It is found that WH fibers as renewable materials can be used to manufacture useful composites with good thermal stability and mechanical properties. The following conclusions could be drawn from the experimental results of this study:

1. Raw, (Anl)/NaOH, and (NNDMA)/NaOH) treated $\mathrm{WH}(35 \mathrm{wt} \%)$ fiber reinforced PP composites are thermally stable up to 273

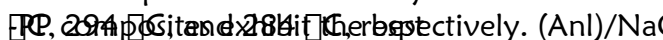
thermal properties in terms of thermal stability and lowest derivative weight loss with respect to time.

2. The tensile strength of the WH-PP biocomposites decreased with an increase in the WH fiber loading. However, the treatment of fibers with $(\mathrm{Anl}) / \mathrm{NaOH}$ gives the maximum tensile strength of the composites. Thus, the treatment of $\mathrm{WH}$ fibers with $(\mathrm{Anl}) / \mathrm{NaOH}$ is recommended when strength is the main concern.

3. In all the cases of composites, the Young's modulus, flexural strength, flexural modulus, and Charpy impact strength are found to increase with an increase in fiber loading and the values are found to be higher for treated WH-PP composites than those of the untreated ones. It is concluded that interaction between the fiber and the matrix has become more favourable upon chemical treatment of WH fibers.

4. Chemical treatment resulted in lower water absorption for treated WH-PP composites compared to raw WH-PP composites. This indicates that hydrophilic nature of WH fiber was reduced upon chemical treatment of WH fiber with Benzenediazonium salt in basic medium.

5. Morphological study of the fracture surface of WH-PP composites shows that, due to favourable interaction between the treated WH fibers and PP, microvoids in the composites are minimized, dispersion of the fibers in the matrix are improved, agglomeration of fibers in the composites and pulling out of fibers are reduced.

The above features ensure the net benefits of the composites of interest of this study. Furthermore, the composites of the present study are biodegradable, cost effective as WH is easily available in nature and environment friendly. Therefore, these composites could be considered as alternatives to costlier wood and can find applications in furniture, door panels, table tops and other potential applications.

\section{ACKNOWLEDGEMENT}

The authors gratefully acknowledge the financial assistance of Bangladesh University of Engineering and Technology. The authors would also like to thank Prof. Monimul Huque, BUET, for his insights into chemical treatment of the fibers and Dr Md Abdul Gafur, BCSIR, Dhaka, for useful discussions while carrying out experiments in BCSIR laboratories.

\section{REFERENCES}

1. Kabir M, Wang H, Lau K, Cardona F. Chemical treatments on plant-based natural fibre reinforced polymer composites: An overview. Composites Part B: Engineering. 2012;43:2883-92.

2. Faruk O, Bledzki AK, Fink H-P, Sain M. Biocomposites reinforced with natural fibers: 2000-2010. Progress in polymer science. 2012;37:1552-96.

3. La Mantia F, Morreale M. Green composites: A brief review. Composites Part A: Applied Science and Manufacturing. 2011;42:579-88.

4. Islam MN, Haque MM, Huque MM. Mechanical and morphological properties of chemically treated coir-filled polypropylene composites. Industrial \& Engineering Chemistry Research. 2009;48:10491-7.

5. John MJ, Thomas S. Biofibres and biocomposites. Carbohydrate polymers. 2008;71:343-64.

6. Edeerozey AMM, Akil HM, Azhar AB, Ariffin MIZ. Chemical modification of kenaf fibers. Materials Letters. 2007;61:2023-5.

7. Islam MS, Hamdan S, Sobuz HR, Rahman R, Ahmed AS. Thermal and decay-resistance properties of tropical wood-plastic composites. Journal of Composite Materials. 2013;47:1493-500.

8. Saha M, Rahman MH, Ali MA. Effect of fiber loadings on mechanical properties of water hyacinth fiber reinforced polypropylene composites. Proceedings of the International Conference on Mechanical Engineering 2011. Dhaka, Bangladesh: ICME11-AM-048; 2011.

9. Bledzki AK, Mamun AA, Volk J. Physical, chemical and surface properties of wheat husk, rye husk and soft wood and their polypropylene composites. Composites Part A: Applied Science and Manufacturing. 2010;41:480-8.

10. Oksman K, Mathew AP, Långström R, Nyström B, Joseph K. The influence of fibre microstructure on fibre breakage and mechanical properties of natural fibre reinforced polypropylene. Composites Science and Technology. 2009;69:1847-53.

11. Haque MM, Hasan M, Islam MS, Ali ME. Physico-mechanical properties of chemically treated palm and coir fiber reinforced polypropylene composites. Bioresource technology. 2009;100:4903-6.

12. Garkhail S, Heijenrath R, Peijs T. Mechanical properties of natural-fibre-mat-reinforced thermoplastics based on flax fibres and polypropylene. Applied Composite Materials. 2000;7:351-72. 
13. Amash A, Zugenmaier P. Morphology and properties of isotropic and oriented samples of cellulose fibrepolypropylene composites. Polymer. 2000;41:1589-96.

14. Supri AG, Ismail $\mathrm{H}$. The effect of NCO-polyol on the properties of low-density polyethylene/water hyacinth fiber (Eichhornia crassiper) composites. Polymer-Plastics Technology and Engineering. 2010;49:766-71.

15. Supri AG, Tan S, Ismail H, Teh P. Effect of poly (methyl methacrylate) modified water hyacinth fiber on properties of low density polyethylene/natural rubber/water hyacinth fiber composites. Polymer-Plastics Technology and Engineering. 2011;50:898-906.

16. Haque M, Islam N, Huque M, Hasan M, Islam S, Islam S. Coir fiber reinforced polypropylene composites: physical and mechanical properties. Advanced Composite Materials. 2010;19:91-106.

17. Kumar A, Singh L, Ghosh S. Bioconversion of lignocellulosic fraction of water hyacinth (Eichhornia crassipes) hemicellulose acid hydrolysate to ethanol by Pichia stipitis. Bioresource Technology. 2009;100:3293-7.

18. Herrera-Franco P, Valadez-Gonzalez A. A study of the mechanical properties of short natural-fiber reinforced composites. Composites Part B: Engineering. 2005;36:597-608.

19. Mir SS, Nafsin N, Hasan M, Hasan N, Hassan A. Improvement of physico-mechanical properties of coirpolypropylene biocomposites by fiber chemical treatment. Materials \& Design. 2013;52:251-7.

20. Islam S, Hamdan S, Jusoh I, Rahman R, Ahmed AS. The effect of alkali pretreatment on mechanical and morphological properties of tropical wood polymer composites. Materials \& Design. 2012;33:419-24.

21. Haque MM, Islam MS, Islam MN. Preparation and characterization of polypropylene composites reinforced with chemically treated coir. Journal of Polymer Research. 2012;19:1-8.

22. Rahman MR, Hasan M, Huque MM, Islam MN. Physico-mechanical properties of jute fiber reinforced polypropylene composites. Journal of Reinforced Plastics and Composites. 2010;29:445-55.

23. Huda M, Drzal L, Misra M, Mohanty A. Wood fiber reinforced poly (lactic acid) composites: Evaluation of the physicomechanical and morphological properties. Journal of Applied Polymer Science. 2006;102:4856-69. 